Korean J. Math. 21 (2013), No. 4, pp. 495-502

http://dx.doi.org/10.11568/kjm.2013.21.4.495

\title{
A REFINED ENUMERATION OF $p$-ARY LABELED TREES
}

\author{
Seunghyun $\mathrm{SeO}^{\dagger}$ And HeEsung Shin ${ }^{\ddagger}$
}

\begin{abstract}
Let $\mathcal{T}_{n}^{(p)}$ be the set of $p$-ary labeled trees on $\{1,2, \ldots, n\}$. A maximal decreasing subtree of an $p$-ary labeled tree is defined by the maximal $p$-ary subtree from the root with all edges being decreasing. In this paper, we study a new refinement $\mathcal{T}_{n, k}^{(p)}$ of $\mathcal{T}_{n}^{(p)}$, which is the set of $p$-ary labeled trees whose maximal decreasing subtree has $k$ vertices.
\end{abstract}

\section{Introduction}

Let $p$ be a fixed integer greater than 1 . A $p$-ary tree $T$ is a tree such that:

(i) Either $T$ is empty or has a distinguished vertex $r$ which is called the root of $T$, and

(ii) $T-r$ consists of a weak ordered partition $\left(T_{1}, \ldots, T_{p}\right)$ of $p$-ary trees.

Received November 29, 2013. Revised December 26, 2013. Accepted December 26,2013

2010 Mathematics Subject Classification: 05A15, 05C05, 05C30.

Key words and phrases: $p$-ary labeled tree, Refinement, Maximal decreasing subtree.

$†$ This research was supported by Basic Science Research Program through the National Research Foundation of Korea (NRF) funded by the Ministry of Education (2011-0008683) and Kangwon National University (120131460).

$\ddagger$ This research was supported by Basic Science Research Program through the National Research Foundation of Korea (NRF) funded by the Ministry of Science, ICT \& Future Planning (2012R1A1A1014154) and INHA University Research Grant (INHA-44756).

$\ddagger$ Corresponding author.

(C) The Kangwon-Kyungki Mathematical Society, 2013.

This is an Open Access article distributed under the terms of the Creative commons Attribution Non-Commercial License (http://creativecommons.org/licenses/by -nc/3.0/) which permits unrestricted non-commercial use, distribution and reproduction in any medium, provided the original work is properly cited. 
A 2-ary(resp. 3-ary) tree is called binary(resp. ternary) tree. Figure 1 exhibits all the ternary tree with 3 vertices. A full p-ary tree is a $p$ ary tree, where each vertex has either 0 or $p$ children. It is well known (see [6, 6.2.2 Proposition]) that the number of full $p$-ary trees with $n$ internal vertices is given by the $n$th order- $p$ Fuss-Catalan number [2, p. 361] $C_{n}^{(p)}=\frac{1}{p n+1}\left(\begin{array}{c}p n+1 \\ n\end{array}\right)$. Clearly a full $p$-ary tree $T$ with $m$ internal vertices corresponds to a $p$-ary tree with $m$ vertices by deleting all the leaves in $T$, so the number of $p$-ary trees with $n$ vertices is also $C_{n}^{(p)}$.

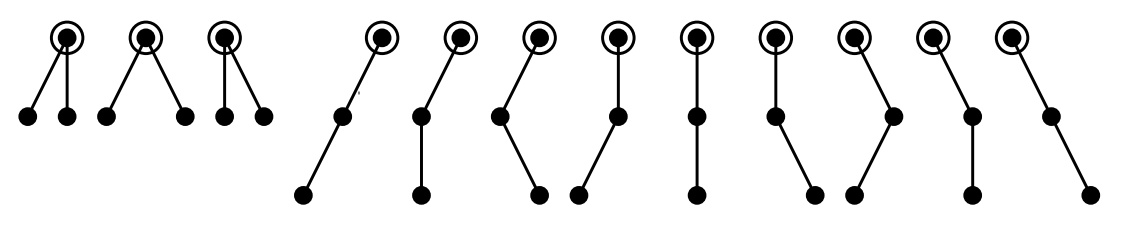

FIgURE 1. All 12 ternary trees with 3 vertices

An $p$-ary labeled tree is a $p$-ary tree whose vertices are labeled by distinct positive integers. In most cases, a $p$-ary labeled tree with $n$ vertices is identified with an $p$-ary tree on the vertex set $[n]:=\{1,2, \ldots, n\}$. Let $\mathcal{T}_{n}^{(p)}$ be the set of $p$-ary labeled trees on $[n]$. Clearly the cardinality of $\mathcal{T}_{n}^{(p)}$ is given by

$$
\left|\mathcal{T}_{n}^{(p)}\right|=n ! C_{n}^{(p)}=(p n)_{(n-1)},
$$

where $m_{(k)}:=m(m-1) \cdots(m-k+1)$ is a falling factorial.

For a given $p$-ary labeled tree $T$, a maximal decreasing subtree of $T$ is defined by the maximal $p$-ary subtree from the root with all edges being decreasing, denoted by $\operatorname{MD}(T)$. Figure 2 illustrates the maximal decreasing subtree of a given ternary tree $T$. Let $\mathcal{T}_{n, k}^{(p)}$ be the set of $p$ ary labeled trees on $[n]$ with its maximal decreasing subtree having $k$ vertices.

In this paper we present a formula for $\left|\mathcal{T}_{n, k}^{(p)}\right|$, which makes a refined enumeration of $\mathcal{T}_{n}^{(p)}$, or a generalization of equation (1). Note that a similar refinement for rooted labeled trees and ordered labeled trees were done before (see $[4,5]$ ), but the $p$-ary case is much more complicated and has quite different features. 


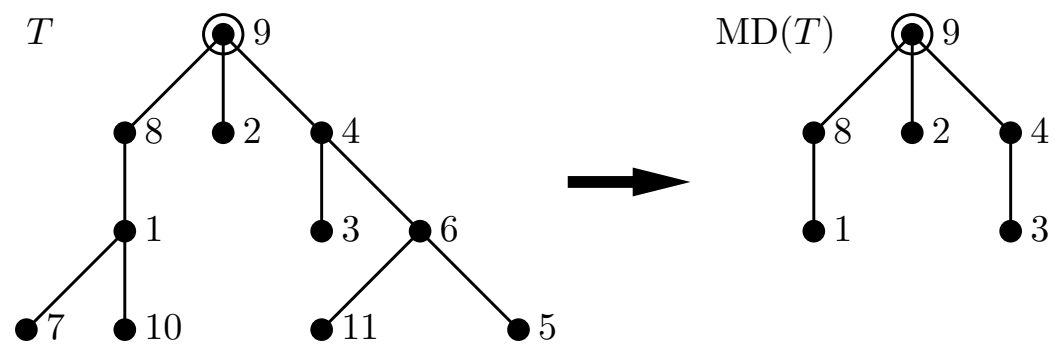

FiguRE 2. The maximal decreasing subtree of the ternary labeled tree $T$

\section{Main results}

From now on we will consider labeled trees only. So we will omit the word "labeled". Recall that $\mathcal{T}_{n, k}^{(p)}$ is the set of $p$-ary trees on $[n]$ with its maximal decreasing ordered subtree having $k$ vertices. Let $\mathcal{Y}_{n, k}^{(p)}$ be the set of $p$-ary trees $T$ on $[n]$, where $T$ is given by attaching additional $(n-k)$ increasing leaves to a decreasing tree with $k$ vertices. Let $\mathcal{F}_{n, k}^{(p)}$ be the set of (non-ordered) forests on $[n]$ consisting of $k p$-ary trees, where the $k$ roots are not ordered. In Figure 3, the first two forests are the same, but the third one is a different forest in $\mathcal{F}_{4,2}^{(2)}$.

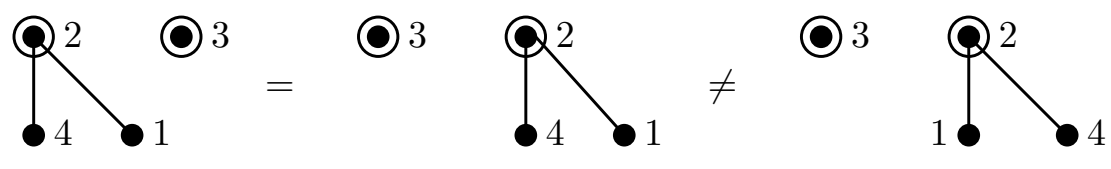

Figure 3. Forests in $\mathcal{F}_{4,2}^{(3)}$

Define the numbers

$$
\begin{aligned}
& t(n, k)=\left|\mathcal{T}_{n, k}^{(p)}\right|, \\
& y(n, k)=\left|\mathcal{Y}_{n, k}^{(p)}\right|, \\
& f(n, k)=\left|\mathcal{F}_{n, k}^{(p)}\right| .
\end{aligned}
$$


We will show that a $p$-ary tree can be "decomposed" into a $p$-ary tree in $\bigcup_{n, k} \mathcal{Y}_{n, k}^{(p)}$ and a forest in $\bigcup_{n, k} \mathcal{F}_{n, k}^{(p)}$. Thus it is important to count the numbers $y(n, k)$ and $f(n, k)$.

Lemma 2.1. For $0 \leq k<n$, the number $y(n, k)$ satisfies the recursion:

$$
y(n+1, k+1)=\sum_{m=0}^{p}\left(\begin{array}{c}
n \\
m
\end{array}\right) p_{(m)}(k p-n+m+1) y(n-m, k)
$$

with the following boundary conditions:

$$
\begin{aligned}
& y(n, n)=\prod_{j=0}^{n-1}(1+(p-1) j) \quad \text { for } n \geq 1 \\
& y(n, k)=0 \quad \text { for } k<\max \left(\frac{n-1}{p}, 1\right) .
\end{aligned}
$$

Proof. Consider a tree $Y$ in $\mathcal{Y}_{n+1, k+1}^{(p)}$. The tree $Y$ with $n+1$ vertices consists of its maximal decreasing tree with $k+1$ vertices and the number of increasing leaves is $n-k$. Note that the vertex 1 is always contained in $\mathrm{MD}(Y)$.

If the vertex 1 is a leaf of $Y$, consider the tree $Y^{\prime}$ by deleting the leaf 1 from $Y$. The number of vertices in $Y^{\prime}$ and $\operatorname{MD}\left(Y^{\prime}\right)$ are $n$ and $k$, respectively. So the number of possible trees $Y^{\prime}$ is $y(n, k)$. Since we cannot attach the vertex 1 to $n-k$ increasing leaves of $Y^{\prime}$, there are $k p-(n-1)$ ways of recovering $Y$. Thus the number of $Y$ with the leaf 1 is

$$
(k p-n+1) \cdot y(n, k) .
$$

If the vertex 1 is not a leaf of $Y$, then the vertex 1 has increasing leaves $\ell_{1}, \ldots, \ell_{m}$, where $1 \leq m \leq p$. Consider the tree $Y^{\prime \prime}$ obtained by deleting $\ell_{1}, \ldots, \ell_{m}$ from $Y$. Clearly 1 is a leaf of $Y^{\prime \prime}$ and the number of vertices in $Y^{\prime \prime}$ and $\operatorname{MD}\left(Y^{\prime \prime}\right)$ are $n-m+1$ and $k+1$, respectively. Thus by (5), the number of possible trees $Y^{\prime \prime}$ is $(k p-(n-m)+1) \cdot y(n-m, k)$. To recover $Y$ is to relabel all the vertices except 1 of $Y^{\prime \prime}$ with the label set $\{2,3, \ldots, n+1\} \backslash\left\{\ell_{1}, \ldots, \ell_{m}\right\}$ and to attach the leaves $\ell_{1}, \ldots, \ell_{m}$ to the vertex 1 of $Y^{\prime \prime}$. Clearly $\ell_{1}, \ldots, \ell_{m}$ is a subset of $\{2,3, \ldots, n+1\}$. It is obvious that a way of attaching $\ell_{1}, \ldots, \ell_{m}$ to vertex 1 can be regarded as an injection from $\ell_{1}, \ldots, \ell_{m}$ to $[p]$. Thus the number of $Y$ without the 


\begin{tabular}{c|rrrrrrrrrr}
$n \backslash k$ & 0 & 1 & 2 & 3 & 4 & 5 & 6 & 7 & 8 & 9 \\
\hline 0 & 1 & & & & & & & & & \\
1 & 0 & 1 & & & & & & & & \\
2 & 0 & 2 & 2 & & & & & & & \\
3 & 0 & 2 & 10 & 6 & & & & & & \\
4 & 0 & 0 & 24 & 56 & 24 & & & & & \\
5 & 0 & 0 & 24 & 256 & 360 & 120 & & & & \\
6 & 0 & 0 & 0 & 640 & 2672 & 2640 & 720 & & & \\
7 & 0 & 0 & 0 & 720 & 11824 & 28896 & 21840 & 5040 & & \\
8 & 0 & 0 & 0 & 0 & 30464 & 196352 & 330624 & 201600 & 40320 & \\
9 & 0 & 0 & 0 & 0 & 35840 & 857728 & 3177600 & 4032000 & 2056320 & 362880
\end{tabular}

TABLE 1. $y(n, k)$ with $p=2$

leaf 1 is

$$
\left(\begin{array}{c}
n \\
m
\end{array}\right)\left(\begin{array}{c}
p \\
m
\end{array}\right) m !(k p-(n-m)+1) \cdot y(n-m, k) .
$$

Since $m$ may be the number from 1 to $p$ and substituting $m=0$ in (6) yields (5), we have the recursion (2).

Since $\mathcal{Y}_{n, n}^{(p)}$ is the set of decreasing $p$-ary trees on $[n]$, the equation (3) holds (see [1]). If the inequality $p k-(k-1)<n-k$ holds, $\mathcal{Y}_{n, k}^{(p)}$ should be empty. For $n \geq 1$ and $k=0, \mathcal{Y}_{n, k}^{(p)}$ is also empty. Thus the equation (4) also holds.

The table for $y(n, k)$ with $p=2$ is shown in Table 1 .

Now we calculate $f(n, k)$ which is the number of forests on $[n]$ consisting of $k p$-ary trees, where the $k$ components are not ordered. Here we use the convention that the empty product is 1 .

Lemma 2.2. For $0 \leq k \leq n$, we have

$$
f(n, k)=\left(\begin{array}{l}
n \\
k
\end{array}\right) p k \prod_{i=1}^{n-k-1}(p n-i) \quad \text { if } n>k,
$$

else $f(n, n)=1$.

Proof. Consider a forest $F$ in $\mathcal{F}_{n, k}^{(p)}$. The forest $F$ consists of (nonordered) $p$-ary trees $T_{1}, \ldots, T_{k}$ with roots $r_{1}, r_{2}, \ldots, r_{k}$, where $r_{1}<r_{2}<$ $\cdots<r_{k}$. The number of ways for choosing roots $r_{1}, r_{2}, \cdots, r_{k}$ from $[n]$ is equal to $\left(\begin{array}{l}n \\ k\end{array}\right)$. From the reverse Prüfer algorithm (RP Algorithm) in [3], 
the number of ways for adding $n-k$ vertices successively to $k$ roots $r_{1}, r_{2}, \cdots, r_{k}$ is equal to

$$
p k(p n-1)(p n-2) \cdots(p n-n+k+1)
$$

for $0<k<n$, thus the equation (7) holds. For $0=k<n, \mathcal{F}_{n, 0}^{(p)}$ is empty, so $f(n, 0)=0$ included in $(7)$. For $0 \leq k=n, \mathcal{F}_{n, n}^{(p)}$ is the set of forests with no edges, so $f(n, n)=1$.

Since the number $y(n, k)$ is determined by the recurrence relation (2) in Lemma 2.1, we can count the number $t(n, k)$ with the following theorem.

Theorem 2.3. For $n \geq 1$, we have

$$
t(n, k)=\sum_{m=k}^{n}\left(\begin{array}{c}
n \\
m
\end{array}\right) \frac{m-k}{n-k}(p n-p k)_{(n-m)} y(m, k) \quad \text { if } 1 \leq k<n,
$$

else $t(n, n)=\prod_{j=0}^{n-1}(p j-j+1)$, where $a_{(\ell)}:=a(a-1) \cdots(a-\ell+1)$ is a falling factorial.

Proof. Given a $p$-ary tree $T$ in $\mathcal{T}_{n, k}^{(p)}$, let $Y$ be the subtree of $T$ consisting of $\operatorname{MD}(T)$ and its increasing leaves. If $Y$ has $m$ vertices, then $Y$ is a subtree of $T$ with $(m-k)$ increasing leaves. Also, the induced subgraph $Z$ of $T$ generated by the $(n-k)$ vertices not belonging to $\operatorname{MD}(T)$ is a (non-ordered) forest consisting of $(m-k) p$-ary trees whose roots are increasing leaves of $Y$. Figure 4 illustrates the subgraph $Y$ and $Z$ of a given ternary tree $T$.

Now let us count the number of $p$-ary trees $T \in \mathcal{T}_{n, k}^{(p)}$ with $|V(Y)|=m$ where $V(Y)$ is the set of vertices in $Y$. First of all, the number of ways for selecting a set $V(Y) \subset[n]$ is equal to $\left(\begin{array}{l}n \\ m\end{array}\right)$. By attaching $(m-k)$ increasing leaves to a decreasing $p$-ary tree with $k$ vertices, we can make a $p$-ary trees on $V(Y)$. So there are exactly $y(m, k)$ ways for making such a $p$-ary subtree on $V(Y)$. Since all the roots of $Z$ are determined by $Y$, by the definition of $\mathcal{F}_{n, k}^{(p)}$ and Lemma 2.2 , the number of ways for constructing the other parts on $V(T) \backslash V(\mathrm{MD}(T))$ is equal to

$$
f(n-k, m-k) /\left(\begin{array}{c}
n-k \\
m-k
\end{array}\right)=\frac{m-k}{n-k}(p n-p k)_{(n-m)} .
$$

Since the range of $m$ is $k \leq m \leq n$, the equation (8) holds. 

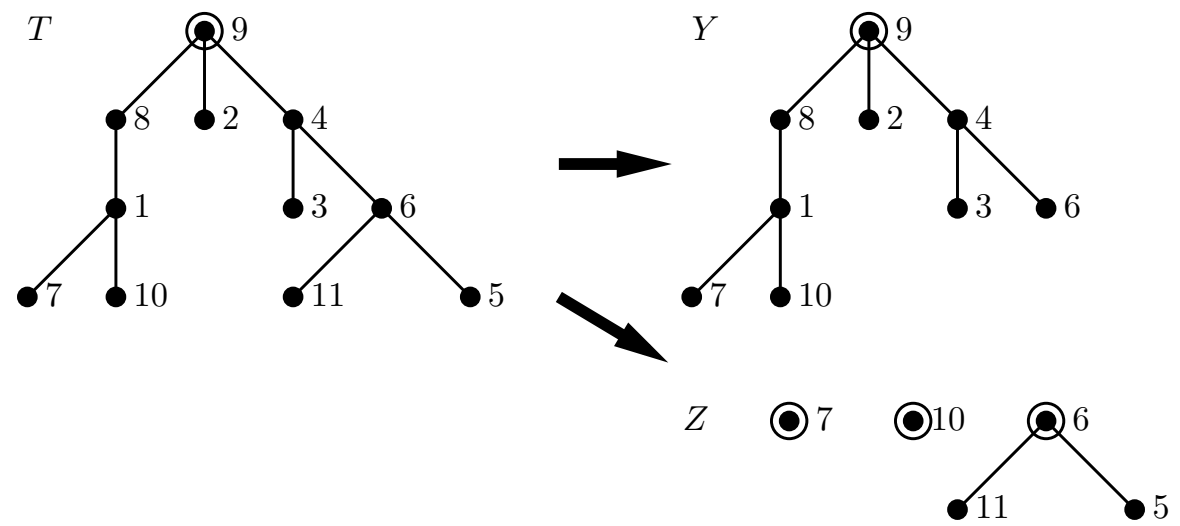

Figure 4. Decomposition of $T$ into $Y$ and $Z$

\begin{tabular}{c|rrrrrrrr|r}
$n \backslash k$ & 0 & 1 & 2 & 3 & 4 & 5 & 6 & 7 & $n ! C_{n}$ \\
\hline 0 & 1 & & & & & & & & 1 \\
1 & 0 & 1 & & & & & & & 1 \\
2 & 0 & 2 & 2 & & & & & & 4 \\
3 & 0 & 14 & 10 & 6 & & & & & 30 \\
4 & 0 & 152 & 104 & 56 & 24 & & & & 336 \\
5 & 0 & 2240 & 1504 & 816 & 360 & 120 & & & 5040 \\
6 & 0 & 41760 & 27744 & 15184 & 6992 & 2640 & 720 & & 95040 \\
7 & 0 & 942480 & 621936 & 342768 & 162240 & 65856 & 21840 & 5040 & 2162160
\end{tabular}

TABLE 2. $t(n, k)$ with $p=2$

Finally, $\mathcal{T}^{(p)}(n, n)$ is the set of decreasing $p$-ary trees on $[n]$, so

$$
t(n, n)=y(n, n)=\prod_{j=0}^{n-1}(p j-j+1)
$$

holds for $n \geq 1$.

The sequence $t(n, k)$ with $p=2$ is listed in Table 2 . Note that each row sum is equal to $n ! C_{n}^{(p)}$ with $p=2$.

Remark. Due to Lemma 2.1 and Theorem 2.3, we can calculate $t(n, k)$ for all $n, k$. In particular we express $t(n, k)$ as a linear combination of $y(k, k), y(k+1, k), \ldots, y(n, k)$. However a closed form, a recurrence relation, or a (double) generating function of $t(n, k)$ have not been found yet. 


\section{References}

[1] François Bergeron, Philippe Flajolet, and Bruno Salvy, Varieties of increasing trees, In CAAP '92 (Rennes, 1992), volume 581 of Lecture Notes in Comput. Sci., pages 24-48. Springer, Berlin, 1992.

[2] Ronald L. Graham, Donald E. Knuth, and Oren Patashnik, Concrete mathematics, Addison-Wesley Publishing Company Advanced Book Program, Reading, MA, 1989. A foundation for computer science.

[3] Seunghyun Seo and Heesung Shin, A generalized enumeration of labeled trees and reverse Prüfer algorithm, J. Combin. Theory Ser. A. 114 (7) (2007), 1357-1361.

[4] Seunghyun Seo and Heesung Shin, On the enumeration of rooted trees with fixed size of maximal decreasing trees, Discrete Math. 312 (2) (2012), 419-426.

[5] Seunghyun Seo and Heesung Shin, A refinement for ordered labeled trees, Korean J. Math. 20 (2) (2012), 255-261.

[6] Richard P. Stanley, Enumerative combinatorics. Vol. 2, volume 62 of Cambridge Studies in Advanced Mathematics. Cambridge University Press, Cambridge, 1999. With a foreword by Gian-Carlo Rota and appendix 1 by Sergey Fomin.

Department of Mathematics Education

Kangwon National University

Chuncheon 200-701, Korea

E-mail: shyunseo@kangwon.ac.kr

Department of Mathematics

Inha University

Incheon 402-751, Korea

E-mail: shin@inha.ac.kr 